\title{
Quantitative Separation of Alkaline Earths Using Diazapolyoxabicyclic Ligands as Ion-size Selective Masking Reagents
}

\author{
Shigekazu TSURUBOU*, Takayuki SASAKI**, Shigeo UMETANI** and Masakazu MATSUI** \\ *Department of Chemistry, School of Dentistry, Asahi University, Hozumi-cho, Gifu 501-02, Japan \\ **Institute for Chemical Research, Kyoto University, Uji, Kyoto 611, Japan
}

\begin{abstract}
Diazapolyoxabicyclic ligands (cryptands) have been applied as ion-size selective masking reagents with the aim of establishing a quantitative extraction-separation system among alkaline earths. Calcium can be quantitatively separated from strontium in the synergistic solvent extraction with 1-phenyl-3-methyl-4-benzoyl-5-pyrazolone (HPMBP) and tri-n-octylphosphine oxide (TOPO), and with benzoyltrifluoroacetone (BFA) and tri-n-butylphosphate (TBP) into cyclohexane in the presence of cryptand[2.2.2] as a masking reagent. Magnesium can also be quantitatively separated from calcium in the synergistic extraction with BFA and TBP into cyclohexane in the presence of cryptand[2.2.1] as a masking reagent.
\end{abstract}

Keywords Diazapolyoxabicyclic ligand, masking reagent, synergistic extraction

Selectivity in solvent extraction of metal ions can be improved by adding the masking reagents to the aqueous phase. The extractability of metal ions with open chain chelating reagents such as $\beta-$ diketons is governed by the stability of the metal complexes. In the solvent extraction of alkali, alkaline earth and lanthanide metal ions with the chelating reagents, metal ions having smaller ionic radii exhibit higher extractability. On the other hand, complex formation of macrocyclic compounds such as crown ethers and cryptands with alkali, alkaline earth and lanthanide cations exhibits a quite different tendency. Thus, a solvent extraction system of high selectivity could possibly be developed by combining the chelating reagents and the macrocyclic ionophores. We have reported that a solvent extraction system of higher selectivity and of quantitative separation can be developed by the combination of chelating reagents and macrocyclic ionophores as ion-size selective masking reagents. ${ }^{1-4}$ By adding the macrocyclic ionophores such as 18-crown-6, 15-crown-5, SB18crown-6 and cryptand[2.2.2] to the aqueous phase as ion size selective masking reagents, the extractions of metal ions with larger ionic radii shifted to the higher $\mathrm{pH}$ region. Consequently, the separation among the metal ions was enhanced.

Diazapolyoxabicyclic ligands (cryptands) exhibit a prominent selectivity for alkali and alkaline earth metals. They are soluble in water and their stability in complexation is large enough for using as a practical masking reagent. Their selectivity for metal ions depends largely on the size of the ring, ${ }^{5}$ and therefore, one can choose an appropriate cryptand according to this purpose.

Quantitative separation between $\mathrm{Ca}$ and $\mathrm{Sr}$ has been achieved in the synergistic extraction with 1phenyl-3-methyl-4-benzoyl-5-pyrazolone (HPMBP) and tri-n-octylphosphine oxide (TOPO) in the presence of cryptand[2.2.2]. In order to utilize the masking effect of cryptands more effectively, benzoyltrifluoroacetone (BFA) has been applied on the synergistic extraction of alkaline earths as a chelating reagent with tri-n-butylphosphate (TBP). By controlling the concentrations of BFA and TBP, quantitative separations between $\mathrm{Mg}$ and $\mathrm{Ca}$, and also between $\mathrm{Ca}$ and $\mathrm{Sr}$ were achieved in the presence of cryptand[2.2.1] and cryptand[2.2.2] respectively as ion-size selective masking reagents.

\section{Experimental}

\section{Apparatus}

The metal ion concentrations in the aqueous phase were determined by an inductivity coupled argon plasma atomic emission spectrometer (Japan Jarrel ash, Model ICAP-500) and an atomic absorption spectrophotometer (Shimadzu, Model AA-660). $\mathrm{pH}$ measurements were made with a Horiba (Model F-22) pH meter after the extraction process. A Taitec (Model BR-30LF) bioshaker was used to equilibrate the aqueous and organic 
phases at a controlled temperature.

\section{Reagents}

HPMBP was synthesized as described in the literature. ${ }^{6}$ The crude compound was recrystallized from dioxane-water and dried under reduced pressure. Analytical data were given in a previous paper. ${ }^{7}$ Cryptand[2.2.1] and cryptand [2.2.2] were purchased from Merck, BFA and TOPO were from Dojindo Laboratories and TBP was from Nacalai Tesque, and used as received. Other chemicals were of analytical-reagent grade. Distilled, and de-ionized water was used throughout.

\section{Procedure}

A $10-\mathrm{ml}$ portion of an aqueous phase containing the required amount of cryptand, metal ion and $0.02 \mathrm{M}$ Good's buffer such as tris(hydroxymethyl)aminomethane was adjusted to the desired $\mathrm{pH}$ with hydrochloric acid and tetramethylammonium hydroxide. The aqueous phase was shaken for 3-20 hrs until the reaction reached to the equilibrium with an equal volume of an organic phase (cyclohexane) containing the required amount of HPMBP and TOPO, or BFA and TBP at $25 \pm 1{ }^{\circ} \mathrm{C}$. After centrifugation of the mixture, the $\mathrm{pH}$ of the aqueous phase was measured. The metal concentration in the aqueous phase was determined by atomic absorption spectrometry and in the organic phase was similarly measured after back-extraction with a hydrochloric acid solution.

\section{Results and Discussion}

Extraction of alkaline earths with HPMBP and TOPO in the absence and presence of cryptand [2.2.2]

Figure 1 shows the results for the extraction of magnesium, calcium, strontium and barium with $0.01 \mathrm{M}$ HPMBP and $0.01 \mathrm{M}$ TOPO into cyclohexane in the absence (blank symbols) and presence (solid symbols) of $0.01 \mathrm{M}$ cryptand[2. 2.2]. Logarithm of the distribution ratio of metal ions between the organic and the aqueous phase, $D$, is plotted against the $\mathrm{pH}$. The slopes of the straight portion of the plots are two, indicating that two protons are released through the extraction reaction. The extraction behaviors for magnesium and calcium in the presence of cryptand[2.2.2] are quite similar to those in the absence of cryptand[2.2.2]. The $\log \mathrm{D}$ values for strontium in the presence of cryptand[2.2.2] increase as the $\mathrm{pH}$ increases similarly to those in the absence of cryptand[2.2.2], then begin to decrease over $\mathrm{pH} 5$. The $\log \mathrm{D}$ values for barium in the presence of cryptand[2.2.2] deviate from those in the absence of cryptand[2. 2 . 2] over $\mathrm{pH} 4.5$. In the synergistic extraction of alkaline earths $\left(\mathrm{M}^{2+}\right)$ with HPMBP (HA) and TOPO, the extraction constant, $\mathrm{K}_{\mathrm{ex}, \mathrm{s}}$ can be

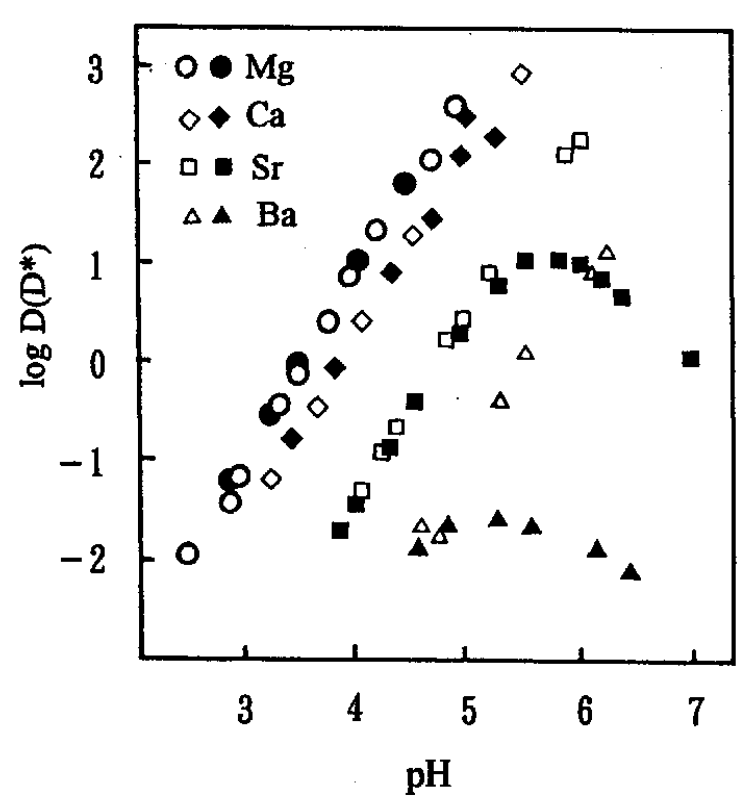

Fig. 1 Extraction of alkaline earths with HPMBP

(HA) and TOPO in the absence (blank symbols) and presence (solid symbols) of cryptand[2.2.2](L). $[\mathrm{HA}]_{0}=[\mathrm{TOPO}]_{0}=1 \times 10^{-2} \mathrm{M}$ in cyclohexane,

$[L]=1 \times 10^{-2} \mathrm{M}$ in an aqueous phase.

written as follows:

$\log \mathrm{Kex}_{\mathrm{s}}=\log \mathrm{D}-2 \mathrm{pH}-2 \log [\mathrm{HA}]_{\mathrm{o}}-\operatorname{slog}[\mathrm{TOPO}]_{\mathrm{o}}$

where subscript $o$ denotes the organic phase and $D$, $[\mathrm{MA2} \text { (TOPO) }]_{\mathrm{O}} /\left[\mathrm{M}^{2+}\right]$, is the distribution ratio of an alkaline earth. The $\log \mathrm{K}_{\mathrm{ex}, \mathrm{s}}$ values were obtained from Fig. 1 and Eq. (1) on the basis of the known $s$ values and are summarized in Table $1 .^{8}$

The distribution ratio of metal ions in the presence of cryptand[2.2.2] (L), D*, can be expressed as follows:

$$
\begin{array}{r}
\left.\mathrm{D}^{*}=\mathrm{Kex}_{\mathrm{s}, \mathrm{HA}}\right]_{\mathrm{o}}{ }^{2}[\mathrm{TOPO}]_{\mathrm{o}}{ }^{\mathrm{s}} / \\
{\left[\mathrm{H}^{+}\right]^{2}\{1+\beta[\mathrm{L}]\}}
\end{array}
$$

where $\beta$ is the complex formation constant of cryptand[2.2.2] with alkaline earths in the aqueous phase, defined as $\left[\mathrm{ML}^{2+}\right] /\left[\mathrm{M}^{2+}\right][\mathrm{L}]$.

$$
\begin{aligned}
& \text { Dividing } \mathrm{D} \text { by } \mathrm{D}^{*} \text { gives } \\
& \qquad \mathrm{D} / \mathrm{D}^{*}=1+\beta[\mathrm{L}]
\end{aligned}
$$

The values of $\log \beta$ were obtained from the straight portion of the plots on Eq. (3) and are summarized in Table 1.

These values are in good agreement with the previously reported data. ${ }^{5}$ Cryptand[2.2.2] is suitable as a masking reagent for calcium and strontium, because the $\log \beta$ values increase in the order, $\mathrm{Mg}<\mathrm{Ca} \ll \mathrm{Sr}<\mathrm{Ba}$, which is the opposite order to that of the conventional chelating reagents. 
The results obtained indicate that the masking effect by cryptand depends on the stability in complexation and on the $\mathrm{pH}$. In order to establish the quantitative extraction-separation system for calcium and strontium, the extraction was made reducing the concentration of HPMBP and TOPO to $4 \times 10^{-3} \mathrm{M}$ keeping the initial concentration of cryptand at $0.01 \mathrm{M}$. As shown in Fig. 2, the distribution ratio of strontium decreases rapidly over $\mathrm{pH} 5.6$ as expected, while that of calcium still increases by $\mathrm{pH} 7.5$. Calcium can be separated from strontium quantitatively at the $\mathrm{pH}$ range 7.2 to 8.6 where more than $99 \%$ of calcium $(\log \mathrm{D}$ $>2$ ) is extracted into cyclohexane, while more than $99 \%$ of strontium $(\log D<-2)$ remains in the aqueous phase at the same time.

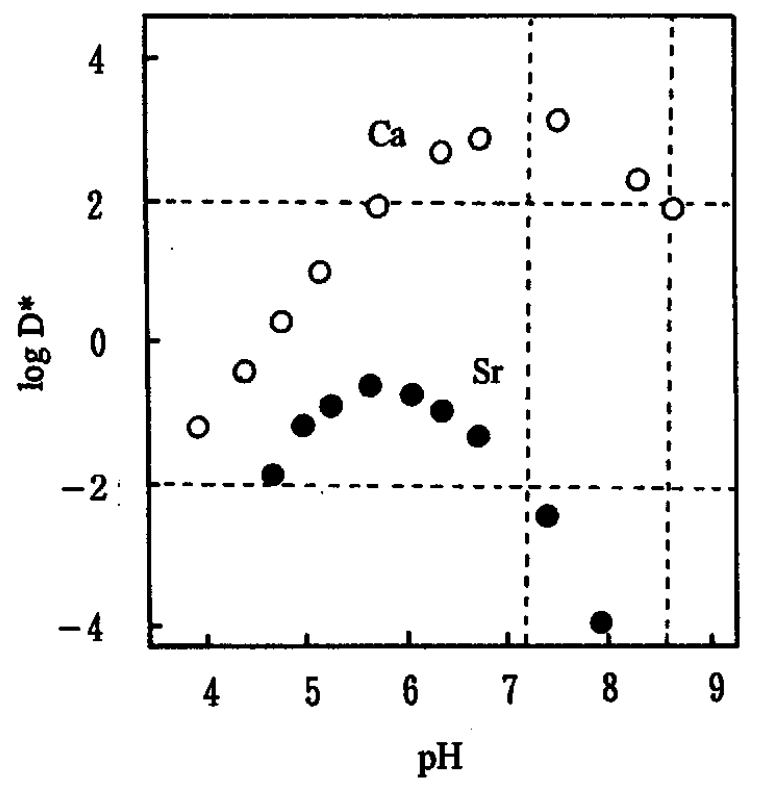

Fig. 2 Extraction of calcium and strontium with HPMBP and TOPO in the presence of cryptand[2.2.2]. $[\mathrm{HA}]_{0}=[\mathrm{TOPO}]_{0}=4 \times 10^{-3} \mathrm{M},[\mathrm{L}]=1 \times 10^{-2} \mathrm{M}$.

Table 1 Extraction constants and stability constants for alkaline earths

\begin{tabular}{lcccc}
\hline & $\log \mathrm{K}_{\text {ex, s }}$ & $\mathrm{s}$ & $\log \beta$ & $\log \beta *$ \\
\cline { 2 - 5 } $\mathrm{Mg}^{2+}$ & 13.45 & 2 & - & $<2$ \\
$\mathrm{Ca}^{2+}$ & 12.85 & 2 & - & 4.4 \\
$\mathrm{Sr}^{2+}$ & 11.19 & 2 & 8.20 & 8.0 \\
$\mathrm{Ba}^{2+}$ & 9.70 & 3 & 9.47 & 9.5 \\
\hline
\end{tabular}

* Taken from Ref. 5.

Extraction of alkaline earths with BFA and TBP in the absence and presence of cryptand[2.2.1] or cryptand [2.2.2]

Because of incorporating nitrogens as an element constituting the ring structure, the masking effect of cryptands depends on a $\mathrm{pH}$ unlike crown ethers such as 18-crown-6. In order to apply cryptands as the masking reagents more effectively, it is desirable the extraction would be done at more alkaline region.

Then, benzoyltrifluoroacetone (BFA) has been applied on the synergistic extraction of alkaline earths as a chelating reagent with tri-n-butylphosphate (TBP).

In the synergistic extraction of alkaline earths $\left(\mathrm{M}^{2+}\right.$ ) with BFA (HA) and TBP, the over-all extraction equilibrium and the extraction constant, $\mathrm{K}_{\mathrm{ex}, \text { s }}$ can be written as same in the synergistic extraction with HPMBP and TOPO as follows:

$\mathrm{M}^{2+}+2 \mathrm{HAo}_{\circ}+\mathrm{sTBP}_{\mathrm{o}} \rightleftarrows \mathrm{MA} 2(\mathrm{TBP})_{\mathrm{s}, \mathrm{o}}+2 \mathrm{H}^{+}$

where subscript $o$ denotes the organic phase. The results for the extraction of magnesium, calcium and strontium with $2.5 \times 10^{-3} \mathrm{M}$ BFA and TBP in cyclohexane are shown in Fig. 3. Alkaline earths were extracted in the order $\mathrm{Mg}>\mathrm{Ca}>\mathrm{Sr}^{9}$ which is the same order in which their ionic radii decrease. Figure 4 shows the results for the extraction of magnesium and calcium into cyclohexane with $2.5 \times 10^{-3} \mathrm{M}$ BFA and TBP in the presence of $0.01 \mathrm{M}$ cryptand[2.2.1]. As shown in Fig. 4, the distribution ratio of calcium decreases rapidly over $\mathrm{pH} 7.5$, while that of magnesium are quite similar to those in the absence of cryptand[2. 2.1].

Magnesium can be separated. from calcium quantitatively at the $\mathrm{pH}$ range $8.5-9.5$ where more than $98 \%$ of magnesium $\left(\log \mathrm{D}^{*}>1.80\right)$ is

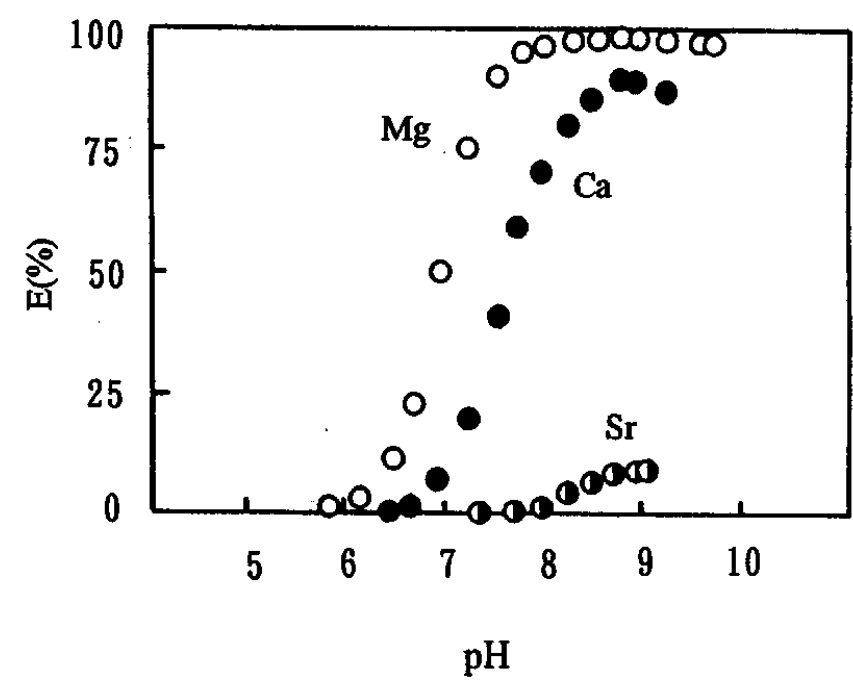

Fig. 3 Extraction of alkaline earths into cyclohexane with BFA and TBP. $[B F A]_{0}=[T B P]_{0}=$ $2.5 \times 10^{-3} \mathrm{M}$.

extracted into cyclohexane, while more than $99 \%$ 


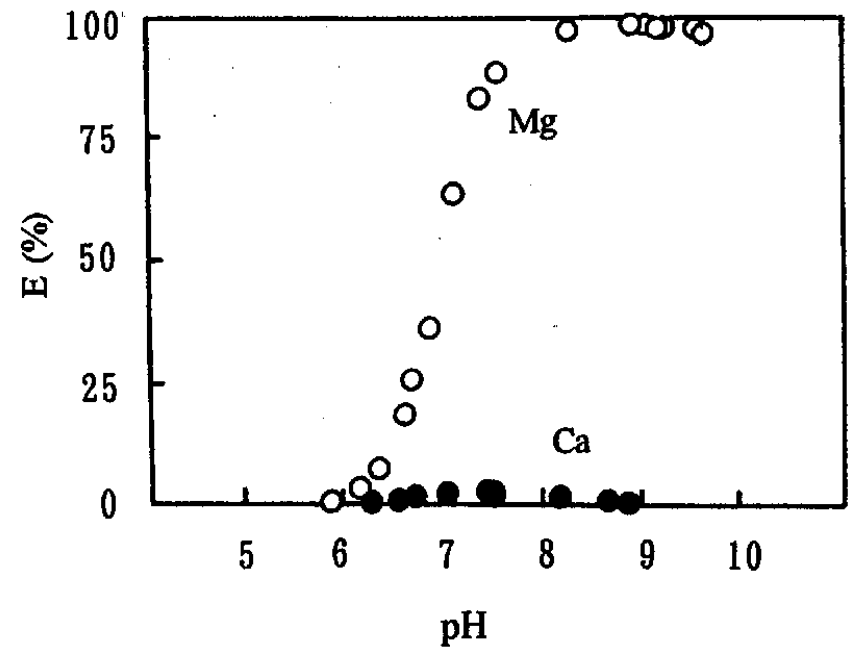

Fig. 4 Extraction of magnesium and calcium into cyclohexane with BFA and TBP in the presence of cryptand[2.2.1].

$[B F A]_{0}=[T B P]_{0}=2.5 \times 10^{-3} \mathrm{M},[\mathrm{L}]=0.01 \mathrm{M}$.

of calcium (log $\left.D^{*}<-2\right)$ remains in the aqueous phase at the same time. On the other hand, the distribution ratios of strontium show the small values $\left(\log \mathrm{D}^{*}<-3\right)$ in the $\mathrm{pH}$ range 7. $0-9.5$.

The $\log \beta$ values of cryptand[2.2.1] were reported to be $<2$ for $\mathrm{Mg}, 6.95$ for $\mathrm{Ca}$ and 7.3 5 for $\mathrm{Sr}^{5}$ The $\log \beta$ value in the present work is in good agreement for $\mathrm{Ca}$. The results obtained indicate that the masking effect of $\mathrm{Ca}$ and $\mathrm{Sr}$ by cryptand[2.2.1] depends on the stability in complexation and on the $\mathrm{pH}$.

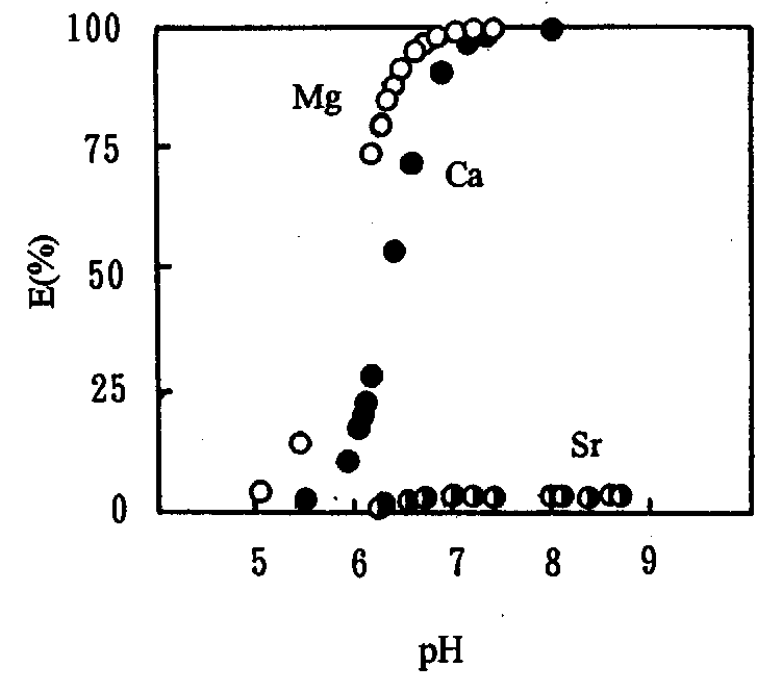

Fig. 5 Extraction of alkaline earths into cyclohexane with BFA and TBP in the presence of cryptand[2.2.2]. $[B F A]_{0}=[T B P]_{0}=0.02 \mathrm{M},[\mathrm{L}]=0.01 \mathrm{M}$.

The distribution ratio of metal ion in the presence of cryptand[2. 2.1], D*, can be expressed as same in the synergistic extraction with HPMBP and TOPO in the presence of cryptand[2. 2. 2] as Eq. (2). The $\log \beta$ value of calcium was obtained from the straight portion of the plots for Eq. (3) to be 7. 0 .

The application of cryptand[2.2.2] to the synergistic extraction of alkaline earths with BFA and TBP has also been examined. Figure 5 shows the results for the extraction of magnesium, calcium and strontium into cyclohexane with $0.02 \mathrm{M}$ BFA and TBP in the presence of $0.01 \mathrm{M}$ cryptand[2.2. 2]. As shown in Fig. 5, very law and constant (about $3 \%$ ) extractability of strontium was observed over $\mathrm{pH} 6.75$, while that of magnesium and calcium are quite similar to those in the absence of cryptand[2. 2. 2]. Strontium can be separated quantitatively from calcium in the $\mathrm{pH}$ range $6.25-$ 8.75 by reducing the concentrations of $\mathrm{BFA}$ and TBP to $0.01 \mathrm{M}$ where more than $99.9 \%(\log \mathrm{D}$ $<-3$ ) of strontium remains in the aqueous phase, while over $98 \%$ of calcium is extracted into cyclohexane with slope of +2 which has the $\mathrm{pH}_{1 / 2}$ value of 6.8. By controlling the concentrations of BFA and TBP, quantitative separations are achieved for $\mathrm{Ca} / \mathrm{Sr}$ in the presence of cryptand[2. 2 . 2] as ion-size selective masking reagent.

The use of the combination of the chelating reagents and diazapolyoxabicyclic ligands, such as cryptand[2.2.1] and cryptand[2.2.2], having an opposite complexation tendency has been proved to be a powerful means to separate alkaline earths.

This concept is universal and is of particular interest because it generates many opportunities for analytical and separation chemistry.

\section{References}

1. S. Umetani, M. Matsui and S. Tsurubou, J. Chem. Soc., Chem. Commun., 914 (1993).

2. T. Sasaki, M. Umetani, M. Matsui and S. Tsurubou, Chem. Lett., 1195 (1994).

3. S. Tsurubou, M. Mizutani, Y. Kadota, T. Yamamoto, S. Umetani, T. Sasaki, Quyen T. H. Le and M. Matsui, Anal. Chem., 67. 1465 (1995).

4. T. Sasaki, S. Umetani, Quyen T. H. Le, M. Matsui and S. Tsurubou, Analyst, 121, 1051 (1996).

5. J. M. Lehn and J. P. Sauvage, J. Am. Chem. Soc., 97, 6700 (1975).

6. B. S. Jensen, Acta Chem. Scand., 13, 1668 (1959).

7. S. Umetani and M. Matsui, Bull. Chem. Soc. Jpn., 56, 3426 (1983).

8. S. Umetani, K. Sasayama and M. Matsui, Anal. Chim. Acta, 134, 372 (1982).

9. R. D. Shannon, Acta Crystallogr., A32, 751 (1976). 